\title{
A 0.7-5.7 GHz Reconfigurable MIMO Receiver Architecture for Analog Spatial Notch Filtering Using Orthogonal Beamforming
}

\author{
Sajad Golabighezelahmad, Student Member, IEEE, Eric Klumperink, Fellow, IEEE and Bram Nauta, Fellow, IEEE
}

\begin{abstract}
A highly reconfigurable direct-conversion softwaredefined MIMO receiver with $4 \mathrm{RF}$ inputs and $4 \mathrm{I} / \mathrm{Q}$ baseband outputs is proposed. It allows for digital MIMO but also analog interference rejection by spatial notch filtering through 4 flexible and simultaneous orthogonal beams. A segmented constant-Gm vector modulator with improved interference tolerance and wide $\mathrm{RF}$ frequency range targeting the sub-6 GHz bands is proposed. It exploits current-domain beamforming before $\mathrm{I}-\mathrm{V}$ conversion by transimpedance amplifiers. A 0.7-5.7 GHz $22 \mathrm{~nm}$ FD-SOI prototype chip achieves $>29 \mathrm{~dB}$ spatial filtering for a single notch and an ultra-wideband $20 \mathrm{~dB}$ notch suppression bandwidth of 2.3 $\mathrm{GHz}$ at broadside excitation at an $\mathrm{LO}$ frequency of $2.5 \mathrm{GHz}$. In the notches, an IIP3 of $+16 \mathrm{dBm}$ and B1dB of $-11.5 \mathrm{dBm}$ at $41 \mathrm{~dB}$ gain is achieved, improving IIP3 and B1dB by $35 \mathrm{~dB}$ and $27 \mathrm{~dB}$, respectively, by spatial filtering. A single-element noise figure of 5.5-7 dB is achieved on the vector modulator constellation corners, degrading about $2 \mathrm{~dB}$ on the points nearby the biggest circle fitting into square constellation. However, sub-3dB system noise figure is potentially achievable taking into account up to $6 \mathrm{~dB}$ improvement by the 4-element beamforming. Given both gain and phase control provided by the vector modulator, spatial patterns with up to 3 independent nulls can be synthesized with the 4-element antenna array. The chip of $0.52 \mathrm{~mm}^{2}$ active area consumes $77-139 \mathrm{~mW}$ at an LO-frequency of 0.7-5.7 $\mathrm{GHz}$ from a 0.8V supply.
\end{abstract}

Index Terms-Spatial filtering, Analog beamforming, MultipleInput Multiple-Output (MIMO), Receiver, Vector Modulator, Interference rejection, Software-defined radio.

\section{INTRODUCTION}

$\mathbf{T}$ HE increasing demand for wireless connectivity is leading to congested radio spectrum especially in the sub$6 \mathrm{GHz}$ bands that are favored over millimeter-wave frequencies for their propagation characteristics as well as energyefficiency. This makes the interference problem a major challenge for wireless systems. To reduce interference, a cognitive radio (CR) can sense spectrum and allocate free channels to users [1]. Moreover, the spatial domain provides other degreesof-freedom to the interference problem. At the receiver side, this can be achieved by canceling out the unwanted signals using spatial filtering.

Multiple antenna systems are needed to leverage spatial signal processing techniques in the analog and digital domains. In analog beamforming phased array receivers [2][8], amplitude and/or phase weighting is applied to multiple antenna signals before summing into a single output that only requires one ADC per array. Analog beamforming is a favorable method because of SNR improvement, its ability to

The authors are with ... cancel out the interferers before entering the ADC, and its ability to improve the interference robustness of the receiver. However, many analog beamforming receivers lack flexibility to realize adaptive beamforming. On the other hand, digital Multiple-Input Multiple-Output (MIMO) systems are capable of more advanced signal processing, achieving an improved data capacity and link reliability for realistic channel models, i.e., propagation reflections and multi-path fading environments [9]. In a digital MIMO receiver, however, element-level analog-to-digital conversion exposes the RF front-ends and following ADCs to strong in-band interferes as shown in Fig. 1a, limiting the dynamic range of the receiver. Adding analog spatial filtering before entering the ADCs can relax dynamic range limits (the baseband implementation is shown in Fig.1b) [10]-[17].
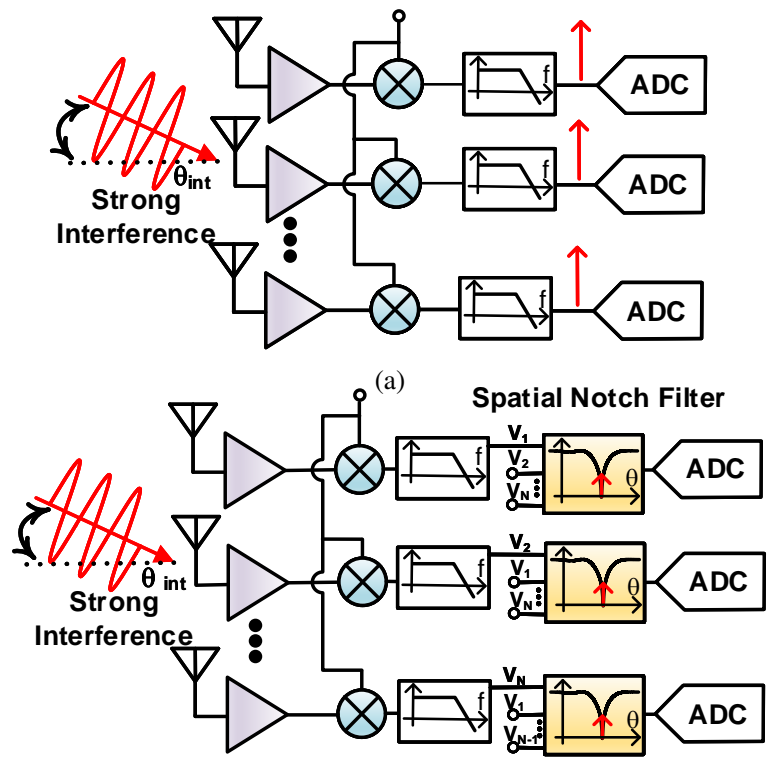

(b)

Fig. 1. (a) A conventional digital MIMO receiver array that is susceptible to a strong co-channel interferer, demanding a high dynamic range ADC; (b) A MIMO receiver incorporating analog spatial notch filtering in the baseband for relaxing the ADC dynamic range [12]-[17].

Only a few research papers have focused on spatial notch filtering techniques for MIMO receivers in sub-6GHz bands [12], [14], [16]. The cancellation at RF, implemented at the LNA output in [11] at $10 \mathrm{GHz}$ requires bulky and bandlimiting quadrature hybrids that makes its implementation less attractive for low-GHz frequencies. A sigma-delta based IF/baseband digital MIMO beamformer was proposed in [18]. 
Reference [19] presents an adaptive beam/null-steering mmwave MIMO Receiver. An analog-FFT based spatio-spectral MIMO Rx was presented in [20]. In [12], a spatial filter realizing one notch in a programmable direction was proposed. The notch is realized in the baseband, after RF amplification and frequency downconversion through a feed-forward canceling circuit, consisting of an angle-independent signal path and a beamformer that contains only the interferer. As linearity and notch rejection bandwidth are limited in [12], reference [14] improves them by canceling interference current to avoid voltage swing at the output of the baseband beamformer. In addition, the synthesis of multiple notches was demonstrated. Although a frequency-translated baseband spatial notch impedance reduces RF gain somewhat, distortion in the RF front-end and active baseband beamformer poses linearity limitations. In [12], [14], a high voltage swing at the LNTA output due to voltage-mode operation of the passive mixer inherently limits the RF frequency range.

In this paper, we describe a new reconfigurable multi-beam architecture supporting both digital MIMO and analog spatial filtering [21]. Spatial interference rejection is achieved using a set of flexible orthogonal beams with a programmable spatialdirection. RF/analog beamforming is realized by an improved constant-Gm vector modulator (VM). In addition to significant linearity enhancement, RF frequency range is extended in a compact and power efficient way, so that the MIMO receiver covers sub- $6 \mathrm{GHz}$ bands in a software-defined radio fashion. Compared to [21], this paper explains the concept in depth, provides analysis of key performance parameters, and adds extra measurement results. Furthermore, the LNTA has been redesigned and a new chip was fabricated to improve the noise performance of the receiver. The next section discusses the proposed receiver architecture. Section III provides the details of circuit implementation, while circuit analysis is presented in Section IV. The experimental results and performance comparison with the state-of-the-art are included in Section V. Finally, Section VI concludes the paper.

\section{PROPOSED INTERFERENCE NULLING RECEIVER}

\section{A. Orthogonal multi-beamforming MIMO receiver architec-} ture

The principle of the proposed MIMO receiver is shown in Fig. 2, where $N$ orthogonal beams are formed for an N-element antenna array and the $N$ ADCs digitize analog beamformed outputs rather than the individual antenna signals as in Fig. 1a. Mathematically, the output beams are independent weighted sums of the input signals. There are as many beams as antenna elements, therefore, they can be used to recover the received signal of each antenna element for further signal processing, providing full digital MIMO functionality. Overall, the system can be considered as a hybrid beamforming system with analog beamforming targeting rejection of the strongest interferers prior to A/D conversion, while the digital part does regular digital MIMO processing. The digital part also adaptively controls the analog rejection, but this will be addressed in a future paper. Here, we demonstrate the interference rejection capabilities of the analog front-end. The

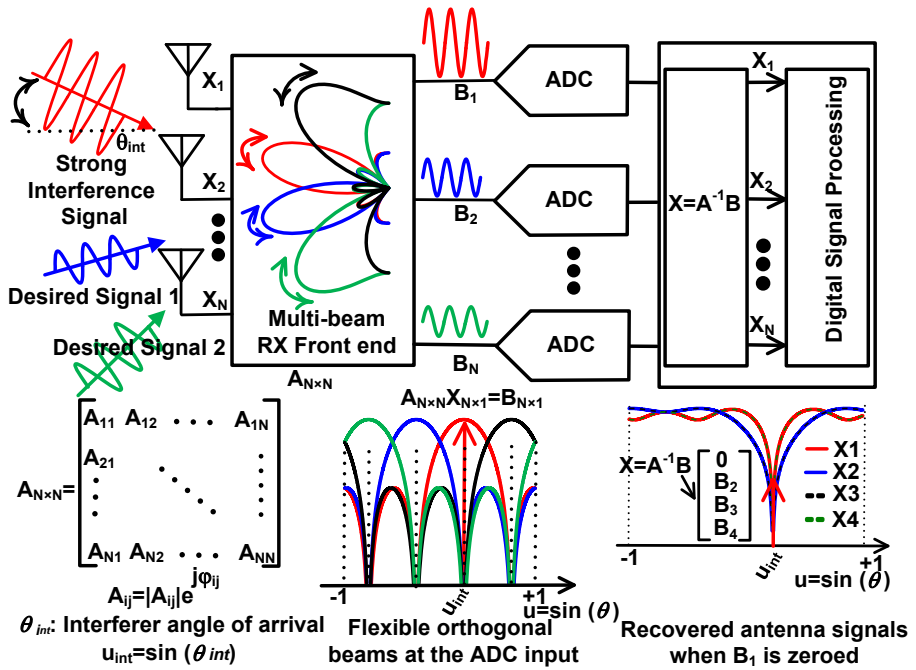

Fig. 2. Proposed analog spatial notch filtering using orthogonal analog beamforming. $N$ Orthogonal beams result for $N$ half-wavelength spaced antennas.

basic idea of the proposed architecture is to separate a strong interferer from a weaker wanted signal, assuming they come from different directions. As illustrated in Fig. 2, this can be achieved using orthogonality, i.e., the beam angle of one output corresponds to the nulls of other outputs (see the lower middle plots in Fig. 2). This feature allows for capturing the interferer in only one beam, e.g. $B_{1}$ in Fig. 2, and reject it at all other remaining outputs, relaxing the dynamic range of all analog circuits after the summing point including the corresponding ADCs. As shown in [22], orthogonality ensures that no information is lost after combining input signals. Hence, orthogonal multi-beamforming being lossless makes it compatible with a MIMO system.

The described interference rejection by orthogonal beamforming is equivalent to analog spatial notch filtering shown in Fig 1b. However, with the proposed method, the notch filtering is achieved by recovering the antenna signals arriving from directions other than the notch angle-of-incidence in the digital domain as shown in the lower right of Fig. 2. This can be done simply by ignoring the heavily distorted output beam containing the interference (e.g., $B_{1}=0$ ). If the inverse of analog preprocessing matrix $\left(A_{N \times N}\right)$ is applied to the ADC outputs, the input antenna signals can be reconstructed while forming a spatial notch in the interferer's angle-of-arrival as shown in the lower right of Fig. 2. Note that this will not make the system 4x3 MIMO (e.g., zero one output with $N=4$ ) and all four antenna signals can be reconstructed in the directions away from $B_{1}$ beam $\left(\theta_{\text {int }}\right)$. The key point here is that $B_{1}$ does not contribute significant useful information in the other directions anyway ( $B_{1}$ has nulls in the main beam directions of $B_{2}, B_{3}$, and $B_{4}$ and "in between" contributes a relatively small part of the signal). So, zeroing $B_{1}$ can admittedly result in some SNR degradation (up to $2.6 \mathrm{~dB}$ with four antenna elements at the sidelobes of $B_{1}$ ), but all $N=4$ antenna signals can be reconstructed in the other directions. Although in a single beamformed output, the main reception angle cannot be arbitrarily steerable if a null angle is fixed, this does not limit the field-of-view for the proposed multi-beam system 
because other outputs can still provide significant signal power. This is evident from the lower right plots in Fig. 2, where the reconstructed antenna signal's spatial patterns are almost flat across the out-of-notch angles. It is also possible to reduce the signal gain (AGC) in the interference beam direction in order to fit it to the ADC dynamic range. This likely comes at the cost of some NF degradation in that direction but it is then at least possible to use that signal digitally. Doing so, the full MIMO capability is still available allowing to gather signals from all directions, avoiding loss of useful signals that might arrive from the interferer direction as well. Overall, this method promises better interference tolerance because each $\mathrm{ADC}$ is only exposed to the interfering signals coming from the direction that analog beams are steered in, while the ADCs in Fig. 1b are more susceptible to interference because of their larger field-of-view.

Several receiver architectures can be envisioned to create a multi-beam phased array receiver. A traditional approach is to use RF multi-beam matrices such as a Butler matrix [23]. A recent CMOS implementation of this passive network can be found in [24]. However, a Butler matrix can only provide fixed beams, lacking the flexibility for arbitrary spatial interference cancellation. While a passive Butler matrix can be highly linear, it consists of bulky passive hybrid couplers that are dedicated for a rather narrow and fixed frequency band and not very CMOS friendly. To overcome these limitations, we propose a flexible multi-beam MIMO receiver architecture employing parallel RF phased arrays as shown in Fig. 3a. The key feature of the proposed architecture is that it performs analog beamfoming directly after V-I conversion by LNTAs in the current-mode domain (see Fig. 3a). This allows for direct interference cancellation at the Trans-Impedance Amplifier (TIA) input before I-V conversion and thus before voltage amplification, resulting in high linearity and blocker-tolerance. Essentially, a virtual ground is provided at the input of TIA which frequency translates to the LNTA output as well, reducing the voltage swing at internal nodes of the receiver (see Fig. 3a). This not only protects the LNTAs against hard compression but also minimizes the distortion induced by the LNTAs and following TIA circuit. Note that we aim to reject a strong co-channel interferer for which spectral filtering is fundamentally absent.

A fully-passive mixer-first realization of the proposed MIMO architecture, as adopted in a single analog beam in [5], can offer even better linearity performance. However, a fully passive architecture does not provide gain, but rather splits in received input signal power to multiple paths. This inherently introduces a noise figure penalty, which is likely unacceptable (4 paths, $12 \mathrm{~dB}$ ).

\section{B. Improved Vector Modulator}

We propose an improved segmented constant-Gm VM [8] as shown in Fig. 3b, to realize amplitude weighting and phase shifting for the MIMO receiver of Fig. 3a. The underlying principle of this power-efficient VM is to slice up an RF frontend consisting of an LNTA followed by a 4-phase passive mixer into $M$ equal slices that are grouped in a binary format, each group of which can contribute either to the I+, Q+, I- or Q- baseband output via a phase selection block implemented by static reconfiguration switches in baseband where parasitic capacitances of the switches are not problematic. This results in a rotated square constellation having $(M+1) \times(M+1)$ points, an example of which is shown for $M=1+2+4=7$, in Fig. 3b.

The main bottlenecks of the constant-Gm VM in [8] are its low RF gain and limited RF frequency range. We have addressed these limitations with the proposed current summing at the TIA input as shown in Fig. 3b, instead of averaging by charge-sharing on the baseband capacitor like in [8]. As the TIA in Fig. 3b provides a low input impedance, the LNTA output node experience a low voltage swing, which results in an extended RF frequency range and improved linearity [25], [26]. Moreover, a higher gain that is not limited to the intrinsic gain of the LNTA can be achieved if a TIA with high feedback resistance is adopted. However, this puts a higher Gain-Bandwidth requirement on the TIA for the virtual ground argument to hold.

For the proposed receiver architecture with four antenna elements, in order to achieve maximum SNR in the absence of strong interferers, the corner points of the constellation (maximum gain) are best used to provide four analog orthogonal beams at $u=0, u=1 / 2, u=-1 / 2, u=1$, where $u=\sin (\theta)$ as shown in Fig. 2, while MIMO functionality can be achieved in the digital domain. In the presence of one strong interference, the equal amplitude points near the biggest circle fitting into the constellation square (Fig. 3b) are best used to form a spatial notch in the interfere's angle-ofincidence as explained above. Although this results in some SNR degradation, in the presence of an interferer, the system performance would mainly be limited by the interferer instead of noise, therefore, the interference cancellation brings much more benefit than the degradation in noise. In the case of multiple interferes, more complex beam patterns with several deep notches are possible, as the proposed VM provides both phase and amplitude control.

\section{Circuit ImplementATiON}

A direct-conversion multi-beam MIMO receiver with 4 $\mathrm{RF}$ inputs and $4 \mathrm{I} / \mathrm{Q}$ baseband outputs is implemented in GlobalFoundries $22 \mathrm{~nm}$ FD-SOI technology as shown in Fig. 4. Each VM provides a complex weight to realize flexible analog beams using $M=15$ slices, which are grouped in a binary format, i.e., $8+4+2+1=15$ to simplify digital control. This results in a $16 \times 16$ points square constellation having 44 equidistant phase points with equal weights on the biggest circle fitting into the constellation points, which leads to a null depth of approximately $29 \mathrm{~dB}$ [8]. An off-chip differential reference signal at twice the LO frequency is used to generate the 4-phase $25 \%$ duty cycle clock signals by an on-chip divideby-two and logic circuits. The resulting quadrature clock signals are distributed throughout the chip to drive the mixer switches. 


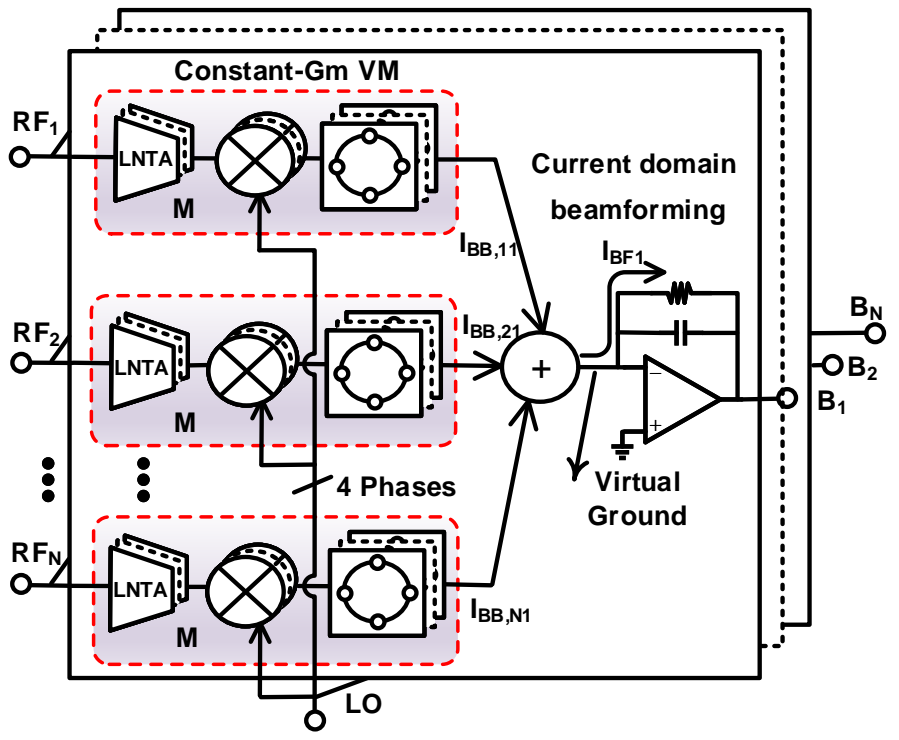

(a)

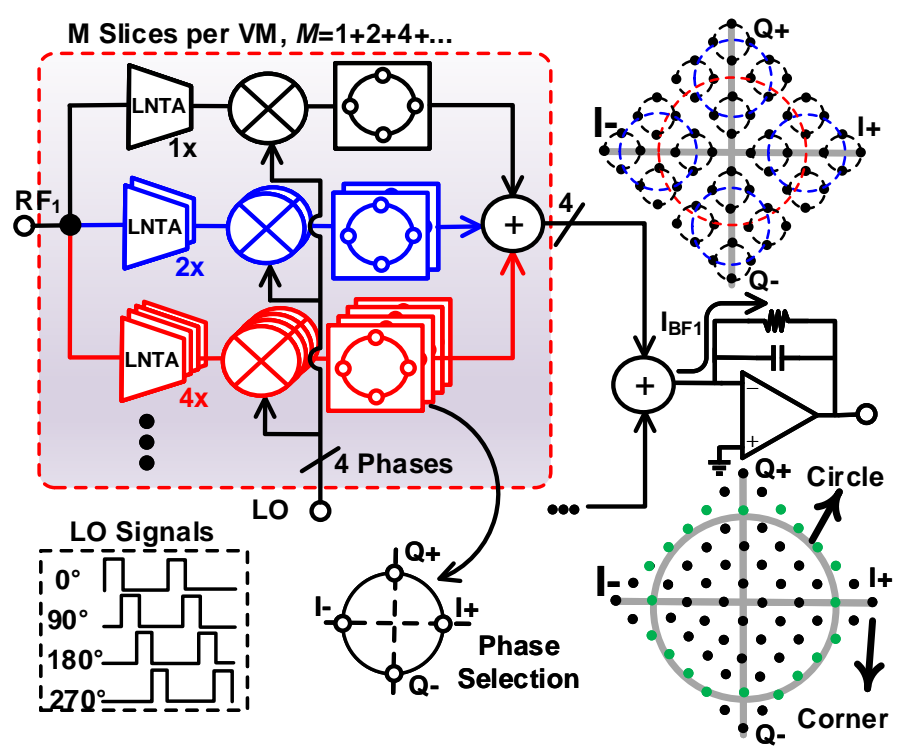

(b)

Fig. 3. (a) Proposed architecture of multi-beam MIMO receiver with multiple RF phased arrays and current domain beamforming; (b) Proposed segmented constant-Gm VM with current summing at the input of TIA. This structure of $M$ slices is repeated $N$ times per beam for beamforming. An example constellation created by a constant-Gm VM with $M=1+2+4=7$ slices $(8 \times 8$ points). The points nearby the biggest circle fitting into the constellation square are used for beam steering in an arbitrary direction.

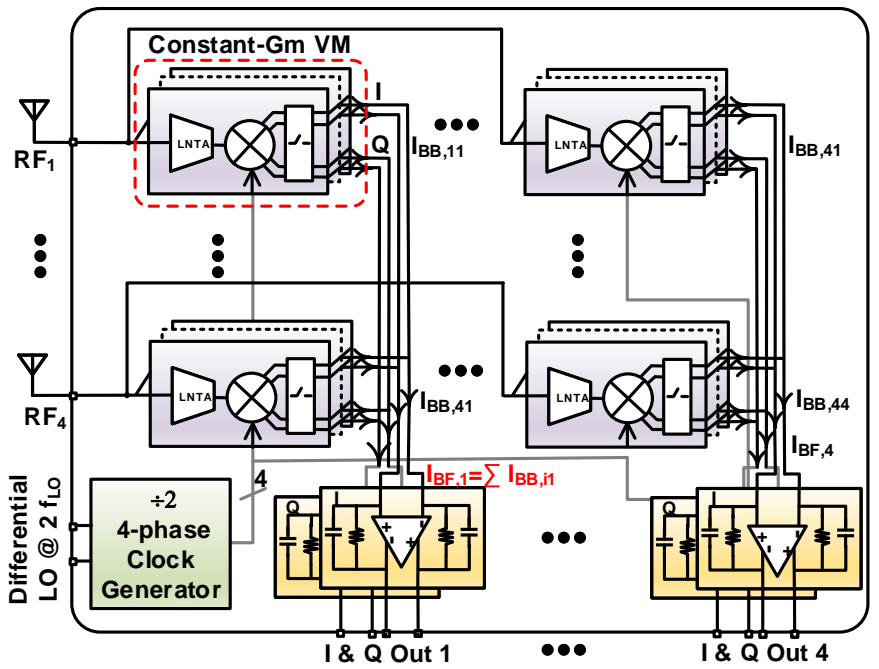

Fig. 4. Circuit implementation of multi-beam $4 \times 4$ MIMO receiver employing 16 constant-Gm vector modulators with current summing (interference rejection) at the input of the TIAs.

\section{A. Unit Slice Circuit}

Figure 5 shows the transistor level implementation for the unit slice of the VM where a self-biased inverter-based LNTA with shunt feedback resistor is followed by a 4-phase passive mixer driven by $25 \%$ duty cycle clock signals. For the LNTA, slightly bigger than minimum-channel-length devices are used to increase the LNTA output resistance as well as minimizing their gate noise. The back gate voltages for both nmos and pmos LNTA transistors are available outside the chip and can be used to tune LNTA's transconductance off-chip if needed during the experiments. These back gates have been always tied to ground for all measurements presented in this paper. The ac coupling capacitor ensures independent and robust
DC biasing of the LNTA and baseband amplifier as well as blocking of second-order intermodulation products created by LNTA. It is realized using Alternative Polarity Metal Oxide Metal (APMOM) capacitor to reduce parasitic capacitances at the RF node. DC level shifting (not shown in Fig. 5) is applied for the LO signals to maximize drive voltage for mixer switches. The on-resistance of the reconfiguration switches is designed to be lower than that of the mixer switches because they do not contribute to dynamic power consumption and their parasitic capacitances are absorbed in the baseband capacitors, thereby not affecting high speed RF performance of the receiver.

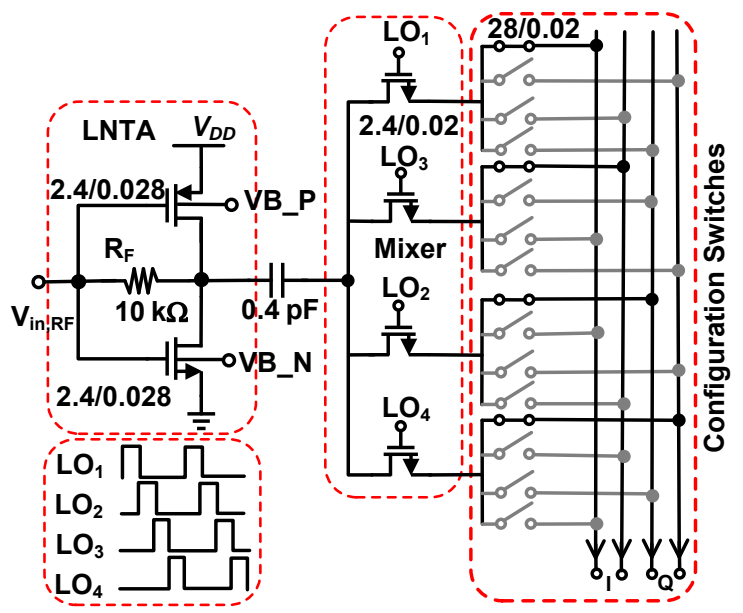

Fig. 5. Unit slice of the VM.

\section{B. TIA Circuit}

The TIA is implemented using self-biased inverter amplifiers employing transistors with large channel lengths $(L=$ 
$0.5 \mu \mathrm{m})$ to achieve high open-loop gain and low flicker noise. Both the feedback resistor and capacitor of the TIA are implemented in a programmable way with 3-bit digital control to provide a variable gain and baseband bandwidth for the receiver (Fig. 6).

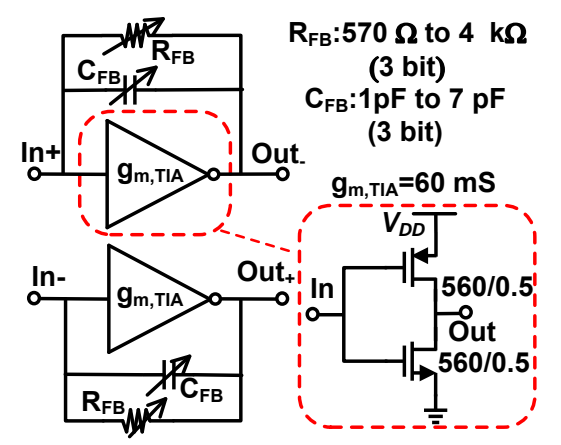

Fig. 6. Implementation of TIA circuit using an inverter amplifier.

\section{CiRCUIT ANAlysis}

In this section, a brief analysis is provided for key performance parameters of the proposed front-end including RF bandwidth extension, conversion gain, input matching, noise, and linearity performance.

\section{A. RF frequency range extension}

As mentioned in Section II, the conventional constant-Gm VM with charge-sharing on a baseband output capacitor [8] (Fig. 7a) poses a limitation in RF frequency range, i.e., inband gain reduction at high RF/LO frequencies. The proposed VM with current summing at the input of the TIA offers wider RF frequency range (Fig. 7b). We will show this by estimating the pole at the LNTA output caused by parasitic capacitance to ground $\left(C_{P}\right)$ and resistance seen in this node for both scenarios. In Fig. 7a, the 4-phase passive mixer operates in voltage-mode. Based on the theory in [27], i.e. $R_{\text {in,mixer }}=R_{\text {sh }} \approx 4.3 R_{\text {out }}$, where $R_{\text {out }}$ is the output resistance of the LNTA. Then, the 3-dB RF cut-off frequency can be found as:

$$
f_{-3 d B, R F}=\frac{1}{2 \pi\left(R_{\text {out }} \| R_{\text {in,mixer }}\right) C_{P}} \approx \frac{1}{2 \pi R_{\text {out }} C_{P}} .
$$

In our design the values of $R_{\text {out }}$ and $C_{P}$ are about $3 \mathrm{k} \Omega$ and $20 \mathrm{fF}$, respectively. Therefore, the maximum achievable RF operating frequency would be limited to $2.7 \mathrm{GHz}$ if chargesharing on a capacitor were to be used. As explained in [28], the capacitance to ground at the RF input of an N-path mixer can have rather complicated effects. According to [28], $C_{P}$ affects the unconverted harmonics of the signal, resulting in a reduction of $R_{s h}$, which reduces in-band gain at high RF/LO frequencies. However, for our circuit, the upconverted baseband impedance is much lower than $R_{s h}$ and the polefrequency at the mixer RF port gives an upper limit for the $\mathrm{RF} / \mathrm{LO}$ frequency at which $3-\mathrm{dB}$ gain reduction occurs. For the proposed VM with current summing (Fig. 7b), the TIA provides a low input impedance $\left(R_{B B}\right)$ that is translated to the RF domain as well. Therefore, if $R_{s h}$ is neglected, the impedance seen at the mixer input is:

$$
\begin{aligned}
& R_{i n, \text { mixer }} \approx R_{s w}+\gamma_{4} N M R_{B B} \\
& R_{B B}=\frac{1}{g_{m, T I A}}+\frac{R_{F B}}{1+A_{v, T I A}}
\end{aligned}
$$

where $\gamma_{4}=\frac{2}{\pi^{2}}$ for a 4-phase passive mixer [27]. $\gamma_{4}$ is related to 4 phases in the passive mixer, so it does not change with the number of antenna elements $N . A_{v, T I A}$ is the openloop gain of the TIA amplifier. $R_{B B}$ in eqn. (2) is scaled by $N M$ because the TIA is shared between $N$ VMs each one having $M$ number of slices that add their currents to the TIA input, thus increasing the voltage swing at both the RF and baseband nodes of the mixer. The value of $R_{\text {in,mixer }}$ is designed to be less than that of $R_{\text {out }}$, thus it dominates the parallel combination of the two resistances, resulting in an RF frequency pole at:

$$
f_{-3 d B, R F}=\frac{1}{2 \pi\left(R_{\text {out }} \| R_{\text {in,mixer }}\right) C_{P}} \approx \frac{1}{2 \pi R_{\text {in,mixer }} C_{P}} .
$$

With the design parameters, e.g., $R_{s w}=150 \Omega, R_{F B}=$ $2 \mathrm{k} \Omega, A_{v, T I A}=300 \mathrm{~V} / \mathrm{V}, M=15, N=4$, and $g_{m, T I A}=$ $60 \mathrm{mS}$, the frequency pole $f_{-3 d B, R F}$ can be extended up to 18 GHz. Circuit simulations with ideal LO clock signals shows that the $3-\mathrm{dB}$ in-band gain reduction due to $C_{P}$ occurs at 13.5 $\mathrm{GHz}$, which is close to the estimated value of the polefrequency. Note that the achievable RF frequency range in practice would be limited by other factors such as the limited rise and fall times of mixer clock edges. However, the resulting limitation due to the charge sharing is resolved to a sufficient degree to allow for operation up to $6 \mathrm{GHz}$. If more antenna elements are used, e.g., an 8 or 16-element array, the RF frequency range still can be improved, however, it requires lower $R_{B B}$ values because $R_{B B}$ in eqn. (2) scales with the number of antenna elements $N$ and the number of slices $M$.

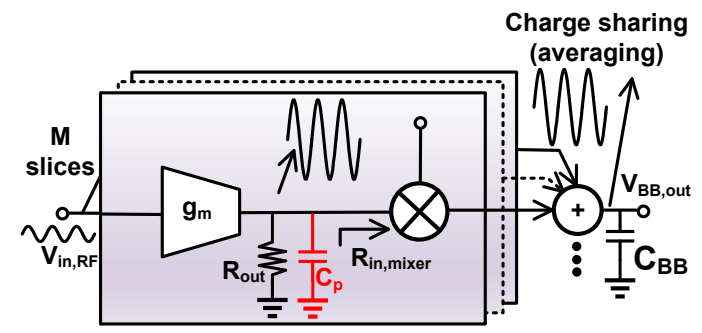

(a)

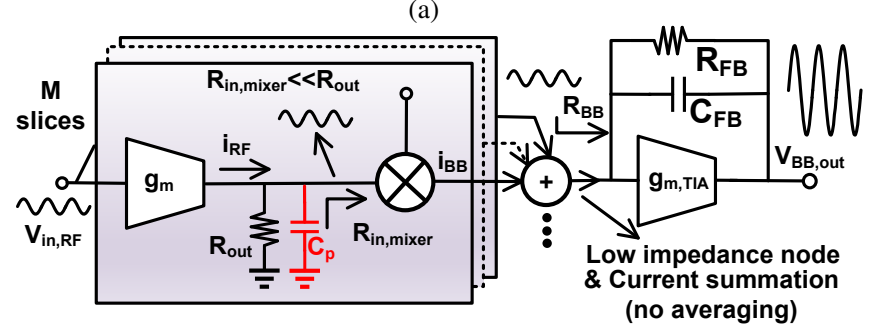

(b)

Fig. 7. (a) Constant-Gm VM with averaging by charge sharing results in high impedance node at the LNTA output and therefore limited RF bandwidth [8]; (b) In the proposed constant-Gm VM with current summing, the RF pole is pushed to higher frequencies by a low-input impedance provided by the TIA. 


\section{B. Conversion gain}

As shown in Fig. 7b, the LNTA converts the RF input voltage to a current, i.e., $i_{R F}=g_{m} V_{i n, R F}$. Assuming that $R_{\text {in,mixer }} \ll R_{\text {out }}$, this current is down converted to the baseband by the $25 \%$ duty cycle passive mixer $\left(i_{B B}=\right.$ $\left.\frac{1}{4} \operatorname{sinc}\left(\frac{1}{4}\right) i_{R F}\right)$. The TIA sums up all the baseband currents from VM slices after which $\mathrm{I}-\mathrm{V}$ conversion across the shunt feedback resistor $R_{F B}$ results in a voltage swing on the baseband output. Therefore, if all the currents are in-phase, i.e., the constellation corners, the differential voltage gain of one VM element can be found:

$$
A_{v, \text { diff }}=\frac{1}{2} \cdot \operatorname{sinc}\left(\frac{1}{4}\right) \cdot M \cdot g_{m} \cdot R_{F B} .
$$

With current summing, the voltage gain scales with the number of slices $M$ as indicated in (5), whereas in the VM of Fig. 7a, the limited intrinsic gain of the LNTA determines the output voltage swing that does not change with the number of slices due to the averaging by charge sharing. Moreover, variable gain control can be realized by a digitally-controlled $R_{F B}$.

\section{Input matching}

Simple inverter-based LNTAs with shunt feedback resistors are used to provide low-noise input matching for the RF inputs. Each antenna is connected to four VMs, where each VM has $M$ slices. Each segmented VM having $M$ slices gives an input impedance that is determined by "Miller"effect as:

$$
R_{i n, V M_{i}}=\frac{1}{M}\left(\frac{R_{F}+R_{L, i}}{1+g_{m} R_{L, i}}\right) \approx \frac{1}{M}\left(\frac{R_{F}}{1+g_{m} R_{L, i}}\right)
$$

where $i=1,2, \ldots, 4$ and $g_{m}$ is transconductance of each LNTA slice and $R_{L, i}$ are load resistors of LNTA slices for corresponding VMs (see Fig. 8a). It is assumed that $R_{F} \gg R_{L, i}$ and the slices of each VM are in-phase, i.e., constellation corners. Neglecting the drain-source output resistance of the LNTA transistors, $R_{L, i}$ are equal to the input impedance of the mixers. Employing an ideal TIA with very small input impedance, each LNTA is only loaded by the on-resistance of the mixer switch. However, with a non-ideal TIA having a finite input impedance, $R_{L, i}$ depend also on the spatial pattern at the TIA inputs. Hence, the input impedance of the VMs varies, accordingly. Actually, the input impedance of the VMs appear to be different for correlated signal than for uncorrelated noise. As we need both values to do beamforming gain calculations on the one hand and noise calculations on the other, we will obtain the input impedance in these example scenarios.

Consider the first scenario with correlated signals, as shown in Fig. 8a, when four orthogonal beams are formed. As the loading impedance of LNTAs depends on the spatial pattern, for the VM looking into the main beam direction, the LNTAs are loaded with $R_{s w}$ in series with the effective translated baseband impedance $\left(\gamma_{4} 4 M R_{B B}\right)$. For the other orthogonal beams nulling occurs (i.e., zero TIA-input current) and the effect of impedance translation is negligible, therefore only $R_{s w}$ remains. The input impedance of each VM can be found using eqn. (6) by substituting the appropriate load impedance for each case and calculating the equivalent impedance of four parallel VMs gives the input impedance for the receiver. Doing this results in the following expression:

$$
R_{i n} \approx \frac{1}{4 M} \frac{R_{F}}{1+g_{m}\left(R_{s w}+\gamma_{4} M R_{B B}\right)}
$$

Note that this input impedance does not depend on the direction unlike the passive beamforming in [5] that provide a low input impedance in the notch direction not matched to $50 \mathrm{ohm}$ load. Note that if a voltage notch at the LNTA output degrades the input matching, this might affect the functionality of a preceding band selection filter. Or unlike notching with baseband impedance synthesis in [14] where the input impedance is higher in the notch direction, potentially degrading interference robustness of the receiver.

In the second scenario, only one input source is excited while the other inputs are resistively terminated by the source impedance (50 ohm here) to model the circuit behavior for uncorrelated input signals, e.g., added noise by each antenna, where the coherent addition of signals from different VM elements does not exist. Fig. $8 \mathrm{~b}$ shows the equivalent receiver circuit model in which all the LNTAs see the same load of $R_{s w}$ in series with $\gamma_{4} M R_{B B}$. Note that in this scenario, the baseband impedance $\left(R_{B B}\right)$ only scales by the number of slices. Therefore, the input impedance is equal to the parallel combination of four equal impedances given by equation (6), which results readily in the same expression as in eqn. (7). Interestingly, both scenarios give the same input impedance. This is because the parallel admittances are simply added to obtain the equivalent admittance and here, the total sum of the LNTA loads are equal for both scenarios, although the individual VMs contribute differently to the input impedance. It can be shown this is also true for the cases in between the two extremes analyzed here. We have verified these results by simulations using a macromodel and circuit implementation.

\section{Noise performance}

To comply with the noise figure (NF) definition in [29] often single-element NF is used to evaluate noise performance of phased array receivers. Here, we will obtain the singleelement noise factor of the proposed beamforming receiver that is defined as the ratio of the total output noise power from one receiver element to the output noise power caused by the source, i.e., antenna noise [29]. To this end, a simplified noise model of one receiver element is shown in Fig. 9. We only take into account the dominant noise contributors, i.e., thermal channel noise of LNTA transconductance, modeled by a current noise $\overline{I_{n, g m}^{2}}=4 k T \gamma g_{m}$ for each slice in Fig. 9, as well as thermal noise of shunt-feedback resistors $\left(\overline{V_{n, R_{F}}^{2}}=4 k T R_{F}\right)$, neglecting the noise caused by the TIA and mixer switches. $R_{i n}$ in Fig. 9 is the input impedance of the receiver and can be calculated from eqn. (7). Although bondwire inductance together with the parasitic input capacitance make a low pass filter in the RF input, for simplicity we do not include its effect in our analysis.

The receiver's noise figure also depends on the VM setting because it affects conversion gain but not the uncorrelated 


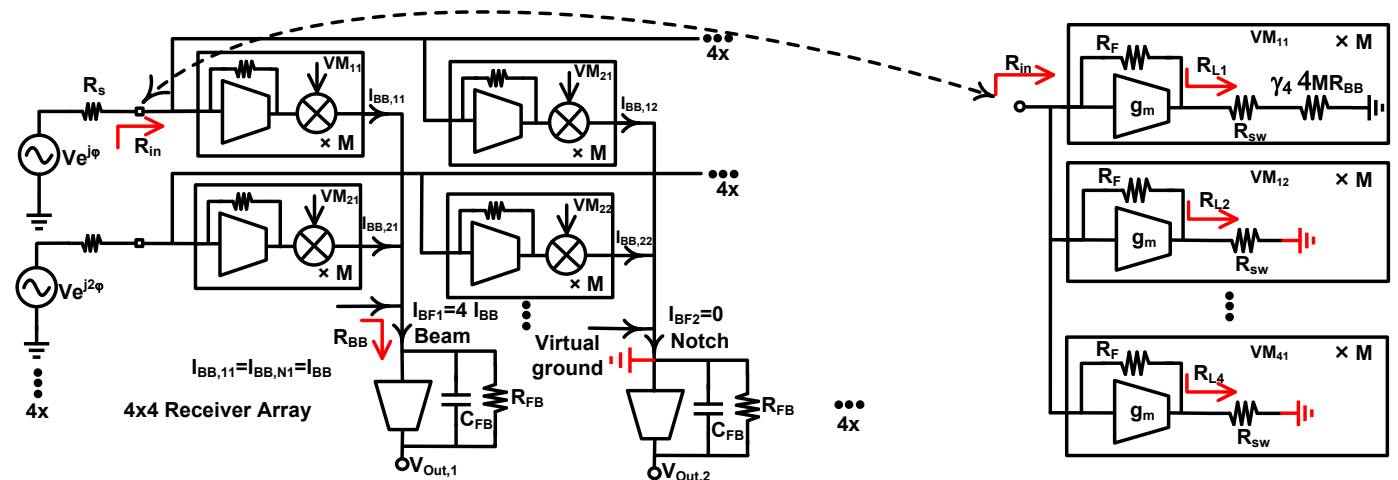

(a)

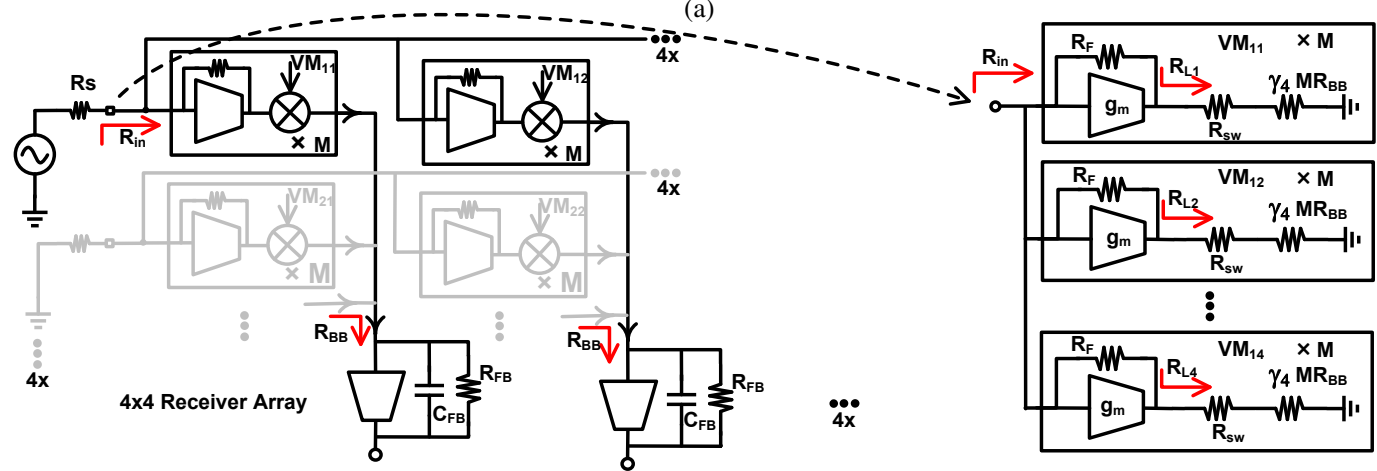

(b)

Fig. 8. Equivalent Circuit model of the input impedance in case of: (a) Correlated signals assuming four orthogonal beams; (b) Uncorrelated input signals (exciting one RF input and terminating the others). Differential I/Q outputs are not shown for simplicity.

added noise of VM slices. To account for this dependency, we define parameter $\alpha$ as the normalized gain of complex constellation points relative to that of the corner point (see Fig. 9). This means that when the VM is set to the corner points $\alpha=1$, and its value is less than one for the other points. More specifically, $\alpha$ is approximately equal to $1 / \sqrt{2}$ for the points near the biggest circle fitting into the constellation square.

Following the above mentioned assumptions, the singleelement noise factor can be obtained as:

$$
\begin{array}{r}
F=\left(1+3 M \frac{R_{s}}{R_{F}}+\frac{1}{\alpha^{2}}\left(\frac{R_{s}+R_{i n}}{R_{i n}}\right)^{2} .\right. \\
\left.\left(\frac{\gamma}{M g_{m} R_{s}}+\frac{\left(1+M g_{m} \frac{R_{s} R_{i n}}{R_{s}+R_{i n}}\right)^{2}}{M g_{m}^{2} R_{F} R_{s}}\right)\right) \frac{1}{\operatorname{sinc}^{2}\left(\frac{1}{4}\right)}
\end{array}
$$

The $3 M R_{S} / R_{F}$ term in (8) is contributed by shunt-feedback resistors in $V M_{2}, V M_{3}$, and $V M_{4}$ (see Fig. 9) that induce noise current into the input node, which appears at the output of element 1 as well. However, this term will only slightly degrade the noise figure if a large value is chosen for $R_{F}$. Due to the low input impedance of the mixers $\left(R_{L} \mathrm{~s}\right.$ in Fig.9) and $R_{F} \gg R_{s}$, the contribution of the $g_{m}$ current noises in $V M_{2}, V M_{3}$, and $V M_{4}$ is negligible, so it is not included in (8). The remaining terms are contributions of $V M_{1}$, scaled by factor $1 / \alpha^{2}$ that represent signal loss due to the VM setting, thereby degrading the noise figure when moving towards the constellation center. As a result, the best noise figure can be achieved at the constellation corner where the gain is maximum, i.e., $\alpha=1$. The $1 / \operatorname{sinc}^{2}(1 / 4)$ term in (8) accounts for harmonic folding noise of a 4-phase passive mixer [26], which adds extra $0.9 \mathrm{~dB}$ to the receiver's noise figure. Using design parameters: $g_{m}=4.2 \mathrm{mS}, R_{F}=10 \mathrm{k} \Omega, R_{s}=50 \Omega$, $R_{i n}=86 \Omega$ from eqn. (7), and assuming $\gamma=2 / 3$ (i.e., long channel assumption for CMOS transistors), equation (8) indicates that a single-element NF of about $3.7 \mathrm{~dB}$ can be achieved in the constellation corner, while it degrades to 5.0 $\mathrm{dB}$ on the circle points. Note, however, that system noise figure benefits from noise averaging for MIMO, bringing up to $6 \mathrm{~dB}$ NF improvement for 4 elements.

The LNTA's in a single-beam $4 \times 1$ array [8] provide higher voltage gain (capacitive output) than the LNTAs terminated with TIAs in this work. This makes some differences in the impedance matching. In an $4 \times 4$ array, we need much larger feedback resistors across LNTAs to meet $50 \mathrm{ohm}$ input matching for more parallel receivers. This is beneficial from a noise perspective, because the higher resistors will contribute less to the noise figure. Also, in an $4 \times 4$ MIMO array, $R_{F} \mathrm{~s}$ from other VMs, e.g., $V M_{2}, V M_{3}$, and $V M_{4}$ connected to the input slightly contribute to noise figure in $V M_{1}$ path as explained above. Overall, there is no significant difference in the noise figure performance between $4 \times 1$ and $4 \times 4$ implementations. Connecting 4 times the number of circuits to the input clearly increase the input parasitic capacitance that degrade the input matching at higher frequencies. However, process scaling helps to reduce this parasitic capacitance, improving the input matching at higher RF frequencies.

\section{E. Linearity and blocker tolerance}

The linearity of a beamforming receiver depends on the angle between interferers and desired signal as was studied in 


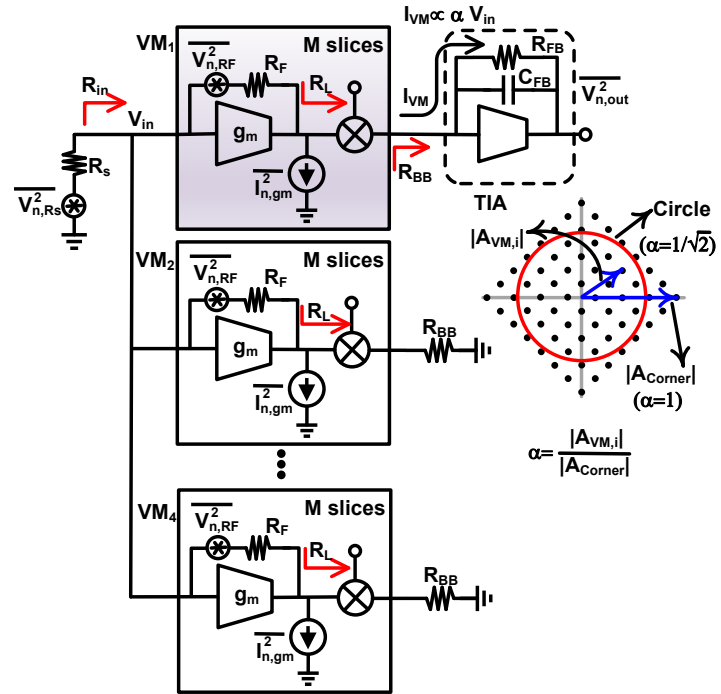

Fig. 9. Single-element noise model of the receiver. For the sake of simplicity, only dominant noise contributors are considered.

[8]. We will now provide some insight into achievable linearity performance of the proposed receiver. For a simple scenario in which a two-tone interferer and a desired signal are both in the main beam direction, it is likely that the last stage, i.e., the TIA limits distortion level at the output. Because of the negative feedback loop as well as the fact that an inverterbased TIA amplifier can provide full swing, a high OIP3 can be achieved. When the two-tone interferer arrives from the notch direction but the desired signal from the main beam angle, if the beamformer is configured to direct a null to the two-tone interferer, the third-order intermodulation (IM3) components created by the non-linear LNTAs is rejected by the spatial notch as well. Note that the TIA processes the interferer residue after spatial filtering occurs, so not only ADC dynamic range but also TIA dynamic range is relaxed. Hence, LNTA nonlinearity rather than TIA nonlinearity tends to dominate distortion. Because with every $2 \mathrm{~dB}$ improvement in IM3, IIP3 improves by $1 \mathrm{~dB}$, the LNTA IIP3 improves by half of the notch depth; e.g., supposing an IIP3 of $+5 \mathrm{dBm}$ for the LNTA and a null depth of $29 \mathrm{~dB}$, in-notch IIP3 of $5+29 / 2=+19.5$ $\mathrm{dBm}$ is expected for the receiver.

An interference nulling receiver has to handle a large cochannel blocker. So, the blocker input power level at which the small signal gain reduces by $1 \mathrm{~dB}(\mathrm{~B} 1 \mathrm{~dB})$ is of interest. As nulling relaxes the dynamic range of the TIA, again the LNTA tends to limit maximum achievable B1dB. For $g_{m}=4.2 \mathrm{mS}$ and $R_{s w}=150 \Omega$ simulation of the simple LNTA circuit shows $\mathrm{B} 1 \mathrm{~dB}$ of $-6.5 \mathrm{dBm}$. Although $\mathrm{B} 1 \mathrm{~dB}$ could be pushed as high as $-3.5 \mathrm{dBm}$ choosing a low-ohmic mixer switch, e.g. $R_{s w}=10 \Omega$ for a unit slice, this would result in a drastically increased dynamic LO power consumption and therefore was not pursued.

\section{EXPERIMENTAL RESUlTS}

A prototype chip was fabricated in GlobalFoundries $22 \mathrm{~nm}$ FD-SOI technology. Fig. 10a shows the chip micrograph. The active area of chip is $0.52 \mathrm{~mm}^{2}$ and a 40-pin QFN package mounted on a four layer PCB, as shown in Fig.10b, was used. Four RF transmission lines on the PCB were designed with equal lengths to minimize phase errors. An external high impedance differential active probe (Lecroy AP033) was used to sense the baseband outputs of the chip.

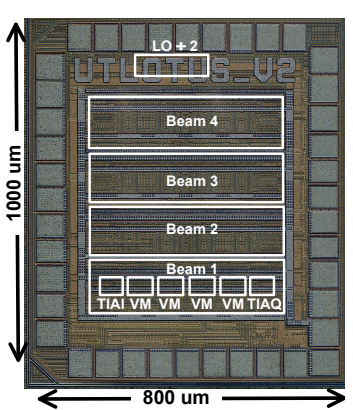

(a)

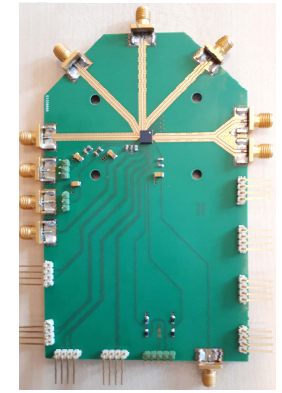

(b)
Fig. 10. (a) Die micrograph; (b) Test PCB with mounted QFN package.

\section{A. Vector modulator and spatial patterns}

The gain and phase accuracy of the implemented VMs is demonstrated with a constellation diagram shown in Fig.12. The constellation was measured with a Vector Network Analyzer (VNA) using a single-tone RF input at LO frequency of $2.5 \mathrm{GHz}$ and compared with an ideal $16 \times 16$ square equidistance constellation. An RMS phase error of $1.3^{\circ}$ and RMS gain error of $0.28 \mathrm{~dB}$ were achieved, demonstrating a close to ideal constellation. The points with equal weights but variable phase nearby the biggest circle fitting into the square constellation are used to create orthogonal beams at arbitrary directions. To measure beam patterns, a 4-element antenna array with uniform half-wavelength spacing is emulated using a commercial 4-way power splitter and four external VMs (TEV2700-45) as shown in Fig. 11. For calibration of the antenna emulation system, first, we have measured complex gains using a VNA across settings of the external vector modulator for four RF paths. Then, we find close to ideal points on a constant gain circle from $1^{\circ}$ to $360^{\circ}$. Later, these data are used during measurements using a lookup table to relate the ideal phases to closest settings. This results in a gain and phase errors of less than $0.2 \mathrm{~dB}$ and $1.5^{\circ}$, respectively, for all of the four paths across $360^{\circ}$ phase points. Therefore, the low gain and phase errors of the measurement set up is sufficient to measure the targeted null depth of about 30 dB. Fig.13a shows an example of orthogonal beam patterns targeting a notch at broadside $\left(u=\sin \left(0^{\circ}\right)=0\right)$ with $f_{L O}=2.5 \mathrm{GHz}$ at $10 \mathrm{MHz}$ baseband. It is seen that a null depth of greater than $29 \mathrm{~dB}$ is achieved. Fig.13a looks similar to what a Butler matrix would provide. However, unlike a Butler matrix with hybrids, here we can flexibly shift the entire pattern, as exemplified in the shifted pattern $(u=0.25)$ in Fig. $13 b$.

To evaluate the notch suppression bandwidth, a notch is formed at broadside and the RF frequency is swept at a fixed LO frequency of $2.5 \mathrm{GHz}$. A $0.25-6 \mathrm{GHz} 4$-way power splitter with low amplitude and phase imbalances (ZN4PD1-63HP+) is utilized to emulate antenna signals arriving from broadside across wide RF frequency range. As shown in Fig.14a, 20 dB 
notch suppression bandwidth is about $2.3 \mathrm{GHz}$. This wideband suppression is possible due to the early current-domain beamforming in the receiver, immediately after Voltage-to-Current conversion by the LNTA and high-linearity current-domainrouting by mixer and reconfiguration switches. Fig.14b shows the same plots as in Fig.14a, but the $\mathrm{x}$-axis represents baseband frequency $\left(f_{B B}\right)$ in logarithmic scale for demonstrating low pass filtering at the baseband output $(\mathrm{BW}=16 \mathrm{MHz})$. Feedback resistor around TIA $\left(R_{F B}\right)$ affects gain, so with higher bandwidth there would be less gain. We have chosen to have a higher gain to show the merits of our receiver better from linearity perspective. Choosing $R_{F B}=2 \mathrm{kohm}, C_{F B}=3 \mathrm{pF}$ results in $41 \mathrm{~dB}$ gain and $16 \mathrm{MHz}$ bandwidth. There is some parasitic capacitance around the TIA amplifier (about $2 \mathrm{pF}$ ), so total feedback capacitance across the TIA amplifier is more than $C_{F B}$. Maximum measured bandwidth of about $100 \mathrm{MHz}$ is obtained in practice.

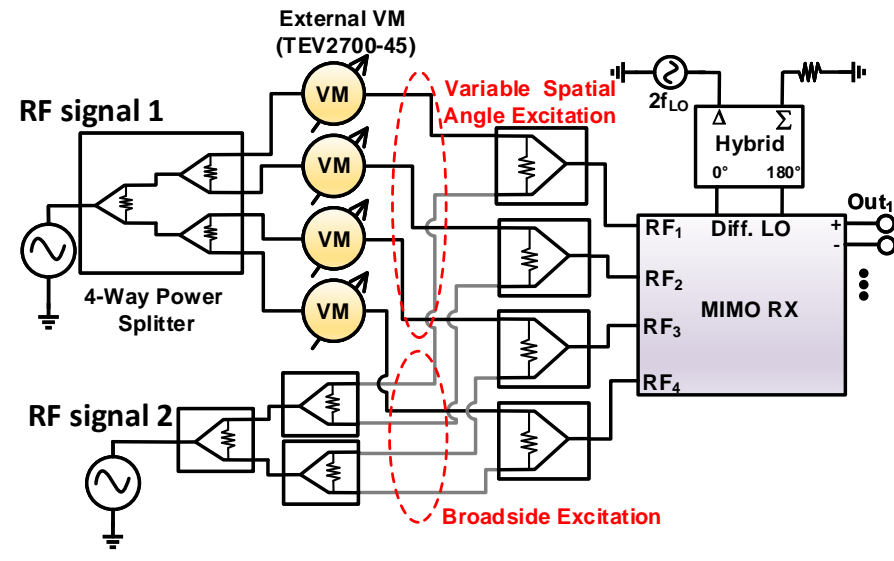

Fig. 11. Block diagram of the 4-element phased array antenna emulation system. Two antenna signals can be emulated one with variable spatial angle and the second one with broadside excitation.

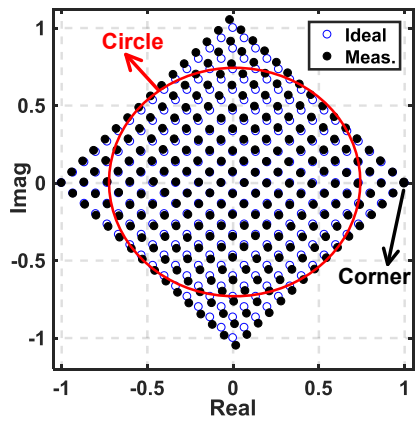

Fig. 12. Measured constellation diagram.

Given that the constant-Gm VM provides both phase and amplitude control, more complex beam patterns with several deep notches can be synthesized using a weight synthesis method to cancel multiple interferers simultaneously. For our 4-element array system, up to 3 independent nulls can be created. Two arbitrary examples of resulting spatial patterns with a main beam pointing to one desired signal direction and three nulls are demonstrated in Fig. 15a and Fig. 15b. A null depths of more than $21 \mathrm{~dB}$ is achieved in both examples.

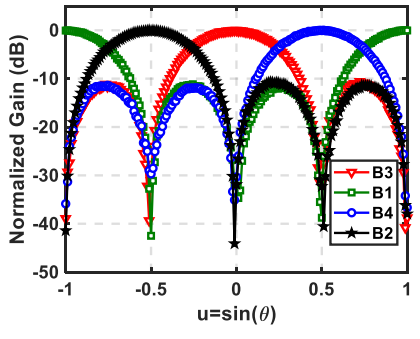

(a)

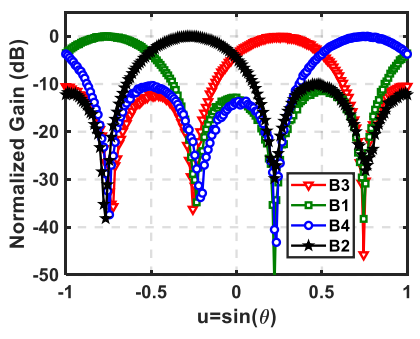

(b)
Fig. 13. (a) Measured orthogonal beam patterns @ $f_{L O}=2.5 \mathrm{GHz}$ with a notch at $\mathrm{u}=0$; (b) Shifted pattern patterns with a notch at $\mathrm{u}=0.25$.

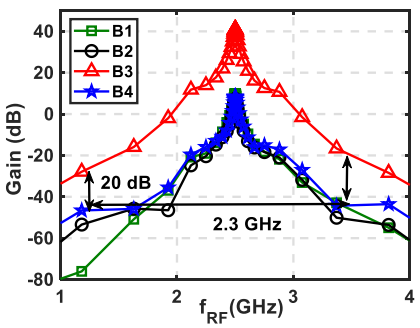

(a)

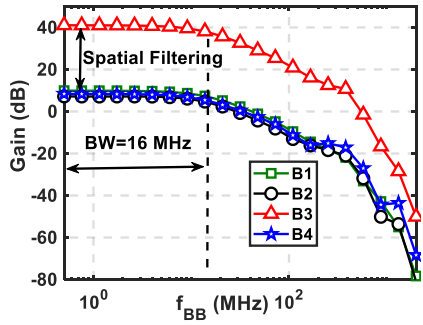

(b)
Fig. 14. $20 \mathrm{~dB}$ notch suppression bandwidth at broadside (a) Gain versus RF frequency; (b) versus baseband frequency, $\mathrm{f}_{\mathrm{BB}}$.

\section{B. Gain, $S_{11}$, and $N F$}

Fig. 16a and Fig. 16b demonstrate widely tunable RFperformance of the chip, by plotting total gain and S11. A $1 \mathrm{MHz}$ IF-tone was used, and the LO frequency was swept. The total gain is measured using the same wideband fourway splitter as in notch bandwidth test, emulating broadside excitation. The plot in Fig. 16a also illustrates wideband spatial filtering (note the difference with Fig. 14a, where RF frequency is swept and LO is fixed, i.e., variable IF-frequency, while here RF and LO are swept together, i.e., fixed IFfrequency). Equivalent single element double sideband noise figure $\left(\mathrm{NF}_{\mathrm{DSB}, \mathrm{eq}}\right)$ versus LO frequency on the constellation corner is shown in Fig. 17a. $\mathrm{NF}_{\mathrm{DSB} \text {,eq }}$ of 6-8.8 dB at broadside $(u=0)$ is measured using the single excitement method introduced by [12], [14], improving the noise performance up to $2.4 \mathrm{~dB}$ compared to the earlier design in [21]. This improvement is achieved by redesigning the LNTAs, i.e., higher $g_{m}$ and slightly higher feedback resistance $\left(R_{F}\right.$ in Fig. 5 ), resulting in only $6 \mathrm{~mW}$ increase in the power consumption. The noise figure varies to some extent depending on the beam

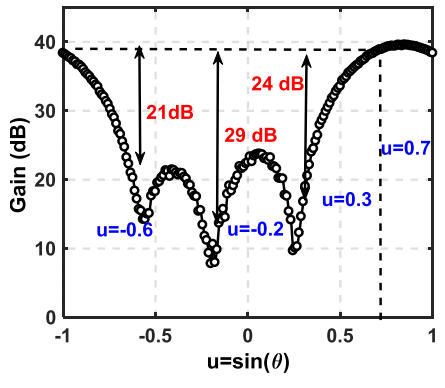

(a)

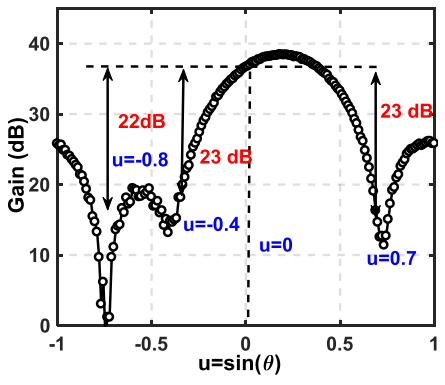

(b)
Fig. 15. (a) An example of synthesized beam pattern targeting a desired signal at $u=0.7$ and three independent nulls at the specified angles; (b) Another example with a desired signal at $u=0$ and other three nulls. 
direction which is mainly due to the gain variations as a result of interaction between RF channels. The noise performance degradation at the low LO frequencies arises from ac coupling capacitors at the LNTA outputs, while the degradation at higher LO frequency most likely is caused by degradation in LO clock signals. The noise figure is also measured on the biggest circle fitting into the square constellation where the signal gain is reduced but analog beams and notches can be steered to arbitrary direction, hence degrading the NF by about $2 \mathrm{~dB}$ (see Fig. 17b). The best achievable system noise figure hence occurs in the corner points (providing analog beams at $\mathrm{u}=0, \mathrm{u}=1 / 2, \mathrm{u}=-1 / 2, \mathrm{u}=1$ and digital beamforming can be performed later at arbitrary direction in the digital domain). When applying digital processing, taking into account the SNR improvement by up to $6 \mathrm{~dB}$, a sub-3dB system noise figure can potentially be obtained. Although the noise figure degrades by moving to circle points, linearity improves much more, i.e. Spurious Free Dynamic Range benefits. Fig. 18 shows the measured noise figure versus baseband frequency at $f_{L O}=2.5 \mathrm{GHz}$. The flicker noise corner is less than 100 $\mathrm{kHz}$.

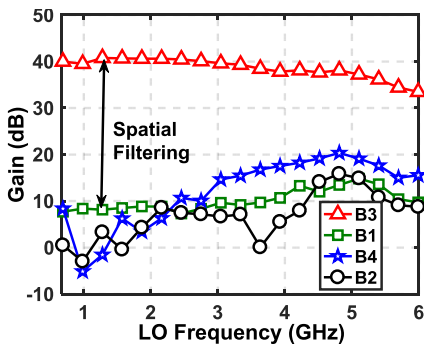

(a)

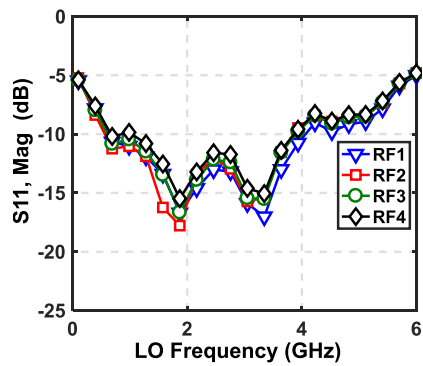

(b)
Fig. 16. (a) Total conversion Gain and (b) $S_{11}$ across LO frequency.

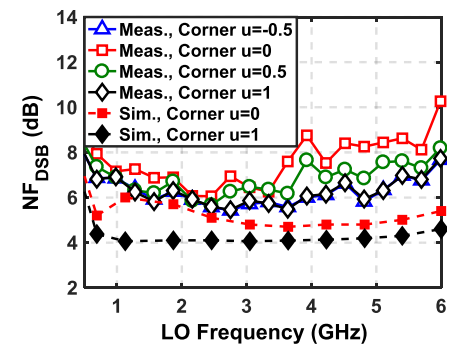

(a)

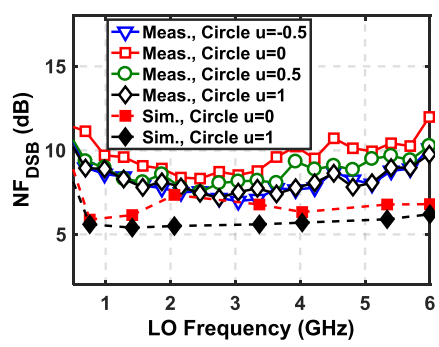

(b)
Fig. 17. (a) Measured and simulated $\mathrm{NF}_{\mathrm{DSB}}$,eq across LO frequency at the constellation corner and (b) $\mathrm{NF}_{\mathrm{DSB}, \mathrm{eq}}$ on the circle points.

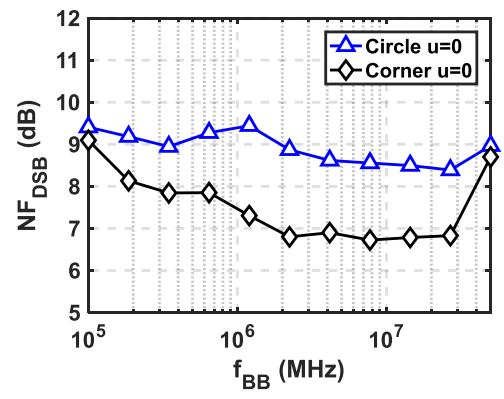

Fig. 18. Measured $\mathrm{NF}_{\mathrm{DSB}, \mathrm{eq}}$ versus $\mathrm{f}_{\mathrm{BB}}$ at $f_{L O}=2.5 \mathrm{GHz}$.

\section{IIP3 and BIdB}

The in-beam/in-notch IIP3 versus offset frequency at $f_{L O}=$ $2.5 \mathrm{GHz}$ and the bandwidth of about $16 \mathrm{MHz}$ is plotted in Fig.19a. The results show $+2 \mathrm{dBm}$ out-of-band linearity in the main beam direction, limited by the linearity of the LNTA. At very low offset frequencies, in-band IIP3 of $-13 \mathrm{dBm}$ and $-19 \mathrm{dBm}$ is measured at a total gain of $35 \mathrm{~dB}$ and $41 \mathrm{~dB}$ respectively, corresponding to OIP3 of $+22 \mathrm{dBm}$ independent from the gain. To evaluate in-notch IIP3, it is assumed that a two-tone interferers arriving at the notch direction have spatial separation from the desired signal angle-of-arrival, e.g., main beam direction. Since the intermodulation product produced by the LNTA is suppressed by the notch filtering, the in-notch IIP3 is improved from +2 to $+20 \mathrm{dBm}$ (at $35 \mathrm{~dB}$ gain) as predicated in section IV and it degrades to $+16 \mathrm{dBm}$ at $41 \mathrm{~dB}$ gain as the nonlinearity of the TIA also starts playing a role at higher gains. This IIP3 remains approximately constant across the offset frequency, which fits to the wide $20 \mathrm{~dB}$ rejection bandwidth observed in Fig.14a. Out-of-band linearity (at offset frequency of about $500 \mathrm{MHz}$ ) versus LO frequency is shown in Fig. 19b. OOB IIP3 improves up to $+6.3 \mathrm{dBm}$ as the LO frequency increases due to reduced LNTA gain because of the lower ac coupling capacitor impedance at higher frequencies. Fig. 20 shows the conversion gain for the desired signal in terms of co-channel, in-beam/in-notch blocker power level; putting a notch at the blocker's angle improves the blocker tolerance about $27 \mathrm{~dB}$, achieving a high $\mathrm{B} 1 \mathrm{~dB}$ of $-11.5 \mathrm{dBm}$.

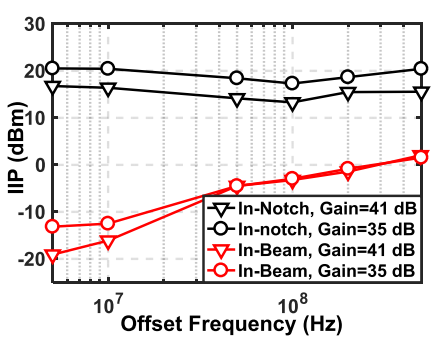

(a)

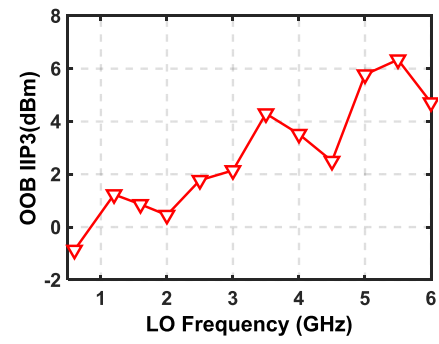

(b)
Fig. 19. (a) In-beam/in-notch IIP3 as function of offset frequency of first tone; (b) Out-of-band IIP3 versus LO frequency

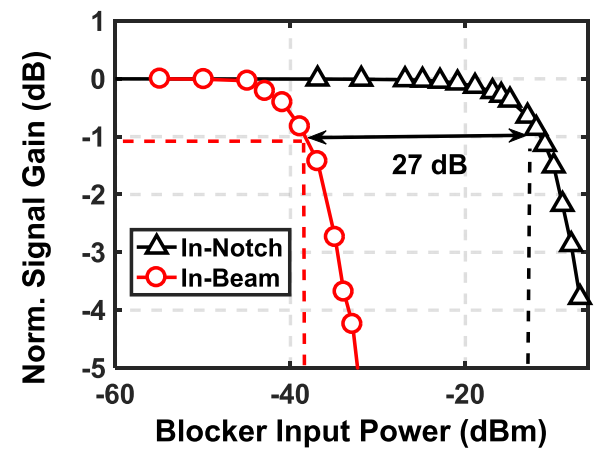

Fig. 20. Conversion gain for desired signal versus co-channel, in-beam/innotch blocker input power

\section{Performance Comparison}

Table I summarizes the performance of the chip and benchmarks it with the state-of-the-art. The receiver is widely 
TABLE I

PERFORMANCE SUMMARY AND COMPARISON WITH STATE-OF-THE-ART.

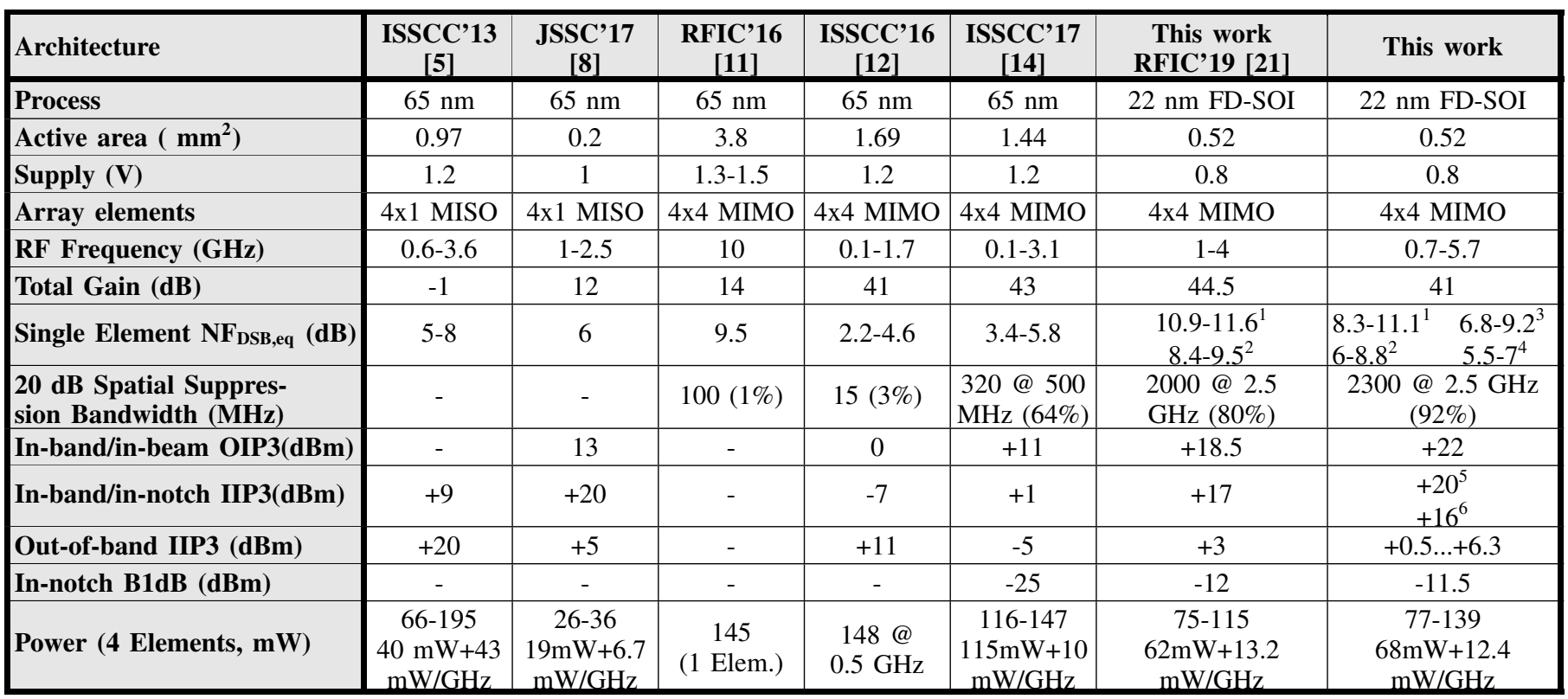

${ }^{1}$ On the circle and broadside $(\mathrm{u}=0)^{2}$ On the corner broadside $(\mathrm{u}=0)^{3}$ On the circle and endfire $(\mathrm{u}=1)^{4}$ On the corner and endfire $(\mathrm{u}=1)$

5 total gain of $35 \mathrm{~dB}^{6}$ total gain of $41 \mathrm{~dB}$

tunable, supporting the sub- $6 \mathrm{GHz}$ bands. The in-beam OIP3 as well as in-notch IIP3 values are the highest among MIMO receivers. Moreover, in-notch $\mathrm{B} 1 \mathrm{~dB}$ of $-11.5 \mathrm{dBm}$ at $41 \mathrm{~dB}$ gain is the best achieved blocker tolerance for MIMO receivers. Taking into account $6 \mathrm{~dB}$ SNR improvement due to the analog beamforming, the receiver can potentially achieve sub$3 \mathrm{~dB}$ noise figure. The noise performance is slightly worse than digital MIMO chips presented in [12], [14], but this is compensated by very significant improvements in linearity. The chip consumes $77-139 \mathrm{~mW}$ at LO frequency of 0.7-5.7 $\mathrm{GHz}$, operating at faster clock speed compared to prior art (about 1.8x), demonstrating a power-efficient circuit implementation. With regard to [21] that consumes $75-115 \mathrm{~mW}$ at LO frequency of $1-4 \mathrm{GHz}$, the static power consumption is slightly increased due to modifications in LNTAs design (about $6 \mathrm{~mW}$ more) and the increase in the higher end is related to the faster clock frequency of $5.7 \mathrm{GHz}$ instead of $4 \mathrm{GHz}$ in [21].

\section{E. EVM measurements}

Measurements were performed to show the effectiveness of spatial filtering using EVM as in [30]. To this end, a setup in Fig. 11 was used where the differential receiver output is sensed using an active probe and applied to a Keysight N9030A PXA Signal Analyzer for digital demodulation by VSA software. To simplify the measurements only the I-output was used. A 10-MSymbols/s 256-QAM desired signal with $50 \mathrm{dBm}$ power level was generated at $f_{R F}=2.47 \mathrm{GHz}$. A root raised cosine pulse shaping filter with $\beta=0.35$ was applied, so the bandwidth of modulated signal is about 13.5 MHz. The baseband bandwidth of the chip was set to 28 $\mathrm{MHz}$ for this experiment. A 5-dB weaker modulated interferer having the same specifications as the desired signal was also generated. The desired signal is arriving from the broadside, while the angle-of-arrival for the interferer can be swept. If the interferer is applied from the same angle as the desired signal, e.g., broadside, digital demodulation fails as shown in Fig. 21a. However, if the interferer angle-of-arrival set to be at the notch angle of $+30^{\circ}$, the desired signal can be successfully demodulated and an EVM of $2.0 \%$ is achieved.

Another EVM measurement was performed while the TIA bandwidth was set to its maximum of about $100 \mathrm{MHz}$. For this experiment, a 50-MSymbols/s 16-QAM desired signal with input power level of $-50 \mathrm{dBm}$ was generated at the center RF frequency $f_{R F}=2.512 \mathrm{GHz}\left(f_{B B}=52 \mathrm{MHz}\right)$. A root raised cosine pulse shaping filter with $\beta=1$ was applied, so the bandwidth of modulated signal is about 100 $\mathrm{MHz}$. A 5-dB stronger modulated interferer having the same specifications as the desired signal was also generated. Output spectrum and constellation with no spatial filtering and after spatial filtering have been shown in Fig. 22a and Fig. 22b, respectively. Digital demodulation fails in the presence of a $5-\mathrm{dB}$ stronger interferer when there is no spatial filtering. However, after $27 \mathrm{~dB}$ interference rejection, an EVM of $8.9 \%$ is achieved $(E V M=-21 \mathrm{~dB})$, which approximately fits to the 22-dB signal-to-interference ratio after spatial filtering. This experiment verifies the wideband spatial interference rejection capabilities of the receiver.

Over-the-air measurements were done using a 4-element dipole antenna array with half-wavelength spacing in the 2.4 $\mathrm{GHz}$ ISM-band, and one dipole antennas for transmitting the desired signal and one antenna for the interferer in the laboratory environment. First, EVM is measured when a 1MSymbols/s 256-QAM desired signal with $-50 \mathrm{dBm}$ input power level and a 20-dB weaker interferer signal having the same modulation specifications as the desired signal are 

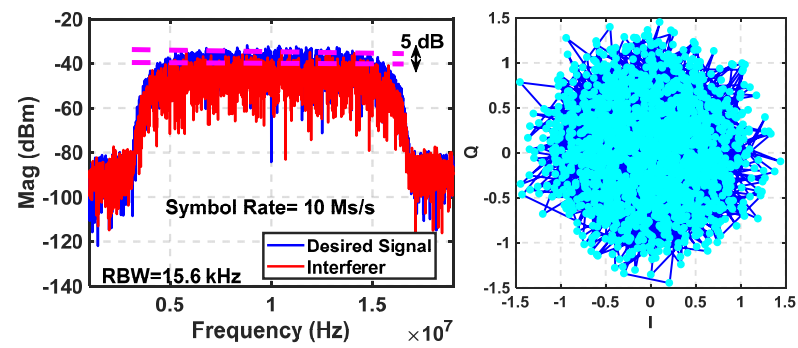

(a)
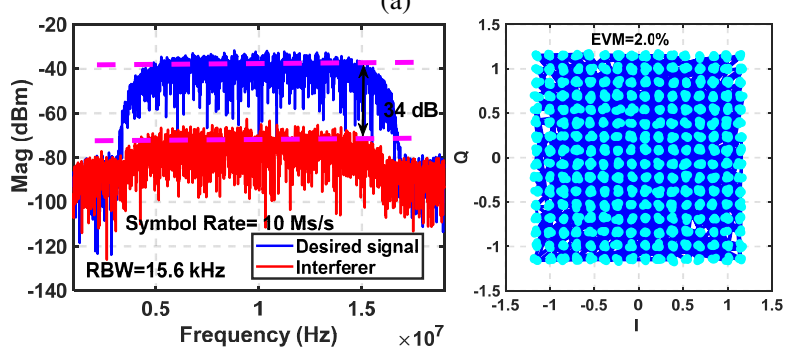

(b)

Fig. 21. (a) Output spectrum and constellation when a modulated desired signal and a 5-dB weaker interferer are both arriving from broadside (no spatial filtering); (b) Output spectrum and constellation when the desired signal is arriving from broadside and the interferer from a null angle at $30^{\circ}$ (spatial filtering).
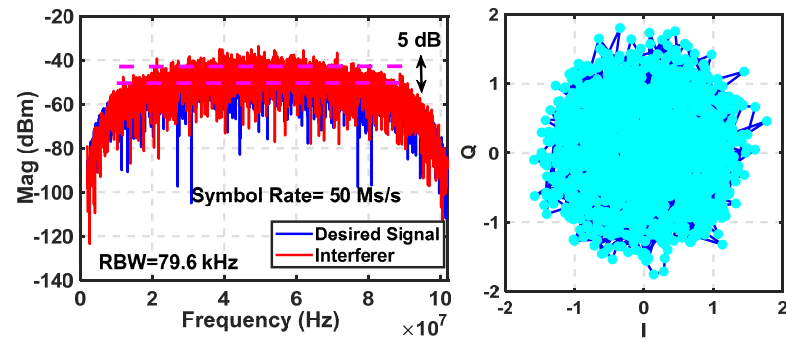

(a)
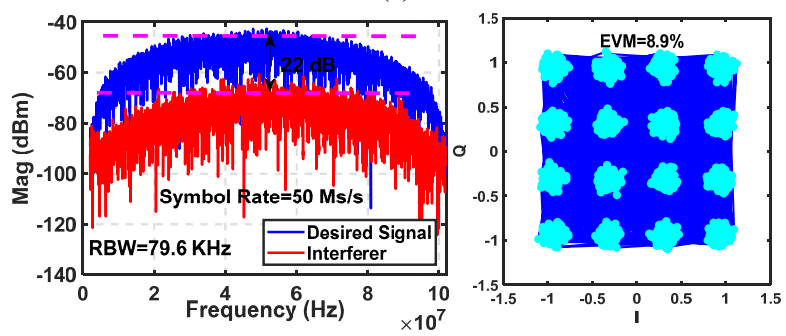

(b)

Fig. 22. (a) Output spectrum and constellation when a 50-MSymbols/s 16 QAM desired signal and a 5-dB stronger interferer are both arriving from broadside (no spatial filtering); (b) Output spectrum and constellation when the desired signal is arriving from broadside and the interferer from a null angle at $30^{\circ}$ (spatial filtering). For this experiment, a root raised cosine pulse shaping filter with $\beta=1$ was used, so the modulated signal bandwidth was about $100 \mathrm{MHz}$.

combined and radiated over-the-air at $2.47 \mathrm{GHz}$ with one antenna placed at broadside. This makes sure that both the interferer and the desired signal propagate to the receiver antenna array within the same propagation channel. As shown in Fig. 23a, digital demodulation has failed. Finally, the interferer again at the same power level as before is transmitted using the second transmit-antenna placed at $+30^{\circ}$ for spatial filtering/separation, after which a successful demodulation becomes possible and an EVM of $1.6 \%$ is obtained as shown in Fig. 23b. With over-the-air experiment, an spatial filtering of $15 \mathrm{~dB}$ is obtained, which is less than that of the conductive measurements because there are variations in the received power at individual antenna array elements possibly due to multi-path propagation as well as mutual coupling between antenna elements, so the results depend on the measurement set-up. However, it is expected that better results become possible utilizing an adaptive spatial filtering solution. We have verified this by manually adapting the beamformer settings. To do so, first the individual antenna signals received from the interferer transmit antenna were measured. This data is used to compensate the amplitude and phase variations relative to expected ideal line-of-sight antenna signals at $30^{\circ}$ by adapting the beamformer weights. This improves the rejection amount to $24 \mathrm{~dB}$.
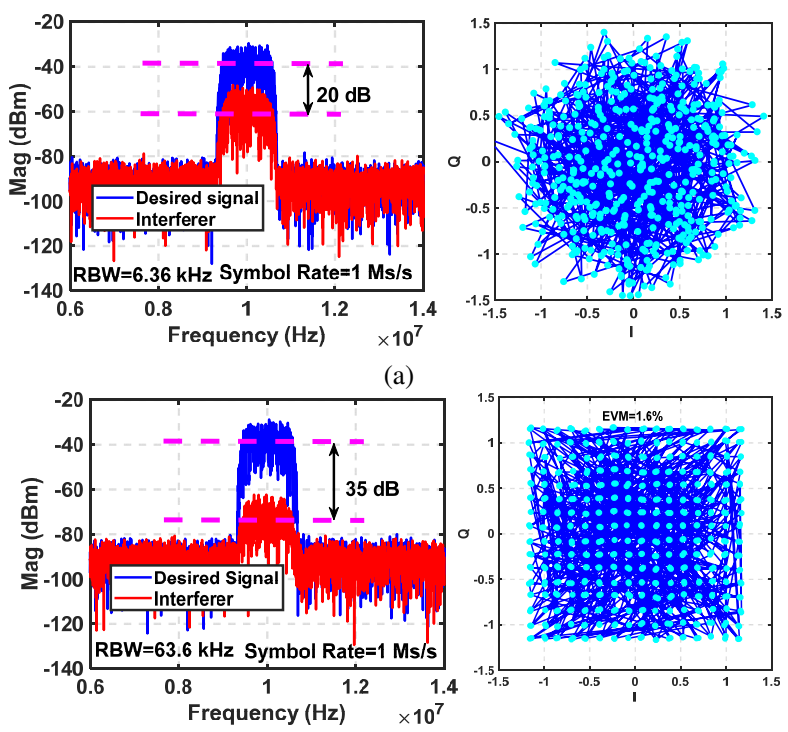

(b)

Fig. 23. (a) Over-the-air measured output spectrum and constellation when a modulated desired signal and a $20-\mathrm{dB}$ weaker interferer are both arriving from broadside (no spatial filtering); (b) Output spectrum and constellation when the desired signal is arriving from broadside and the interferer from a null angle at $30^{\circ}$ (spatial filtering).

\section{CONCLUSION}

In this paper, a highly flexible and reconfigurable MIMO receiver was presented for spatial co-channel blocker rejection. The receiver is tunable up to $6 \mathrm{GHz}$, supporting sub- $6 \mathrm{GHz}$ bands. The main focus of this work is on reconfigurable interference notching MIMO front-end to relax the dynamic range of the baseband amplifier and ADCs. Interference suppression early in the receiver chain before voltage amplification improves linearity performance as well as notch suppression bandwidth. An in-notch IIP3 of $+20 \mathrm{dBm}$ and $+16 \mathrm{dBm}$ at total gain $35 \mathrm{~dB}$ and $41 \mathrm{~dB}$, respectively, and a highly blocker tolerant performance with $\mathrm{B} 1 \mathrm{~dB}$ of $-11.5 \mathrm{dBm}$ at $41 \mathrm{~dB}$ gain are achieved.

\section{ACKNOWLEDGMENT}

The authors would like to thank Gerard Wienk for CAD assistance and Arnoud Rop for measurement setup. The authors also thank GlobalFoundries for supporting chip fabrication. This work is part of the TTW-research program MIRABEAM 
with project number 14689 , which is financed by the Dutch Research Council (NWO).

\section{REFERENCES}

[1] J. Mitola and G. Q. Maguire, "Cognitive radio: making software radios more personal," IEEE Personal Communications, vol. 6, no. 4, pp. 1318, Aug. 1999.

[2] J. Paramesh, R. Bishop, K. Soumyanath, and D. Allstot, "A 1.4V $5 \mathrm{GHz}$ four-antenna Cartesian-combining receiver in 90nm CMOS for beamforming and spatial diversity applications," in IEEE Int. Solid-State Circuits Conf. (ISSCC) Dig. Tech. Papers, vol.1, Feb. 2005, pp. 210-594.

[3] R. Tseng, H. Li, D. H. Kwon, Y. Chiu, and A. S. Y. Poon, "A Four-Channel Beamforming Down-Converter in 90-nm CMOS Utilizing Phase-Oversampling," IEEE J. Solid-State Circuits, vol. 45, no. 11, pp. 2262-2272, Nov. 2010.

[4] M. C. M. Soer, E. A. M. Klumperink, B. Nauta, and F. E. van Vliet, "A 1.0-to-4.0GHz $65 \mathrm{~nm}$ CMOS four-element beamforming receiver using a switched-capacitor vector modulator with approximate sine weighting via charge redistribution," in IEEE Int. Solid-State Circuits Conf. (ISSCC) Dig. Tech. Papers, Feb. 2011, pp. 64-66.

[5] A. Ghaffari, E. A. M. Klumperink, F. E. van Vliet, and B. Nauta, "Simultaneous spatial and frequency-domain filtering at the antenna inputs achieving up to $+10 \mathrm{dBm}$ out-of-band/beam $\mathrm{P} 1 \mathrm{~dB}$," in IEEE Int. Solid-State Circuits Conf. (ISSCC) Dig. Tech. Papers, Feb. 2013, pp. $84-85$.

[6] M. C. M. Soer, E. A. M. Klumperink, B. Nauta, and F. E. van Vliet, "1.0to $-2.5 \mathrm{GHz}$ beamforming receiver with constant-Gm vector modulator consuming $<9 \mathrm{~mW}$ per antenna element in $65 \mathrm{~nm}$ CMOS," in ISSCC, Feb. 2014, pp. 66-67.

[7] S. K. Garakoui, E. A. M. Klumperink, B. Nauta, and F. E. van Vliet, "Compact Cascadable g m -C All-Pass True Time Delay Cell With Reduced Delay Variation Over Frequency," IEEE J. Solid-State Circuits, vol. 50, no. 3, pp. 693-703, March 2015.

[8] M. C. M. Soer, E. A. M. Klumperink, D. van den Broek, B. Nauta, and F. E. van Vliet, "Beamformer With Constant-Gm Vector Modulators and Its Spatial Intermodulation Distortion," IEEE J. Solid-State Circuits, vol. 52, no. 3, pp. 735-746, March 2017.

[9] A. J. Paulraj, D. A. Gore, R. U. Nabar, and H. Bolcskei, "An overview of MIMO communications - a key to gigabit wireless," Proc. IEEE, vol. 92, no. 2, pp. 198-218, Feb. 2004.

[10] J. H. C. van den Heuvel, J. P. M. G. Linnartz, P. G. M. Baltus, and D. Cabric, "Full MIMO Spatial Filtering Approach for Dynamic Range Reduction in Wideband Cognitive Radios," IEEE Trans. Circuits Syst. I, vol. 59, no. 11, pp. 2761-2773, Nov. 2012.

[11] S. Jain, Y. Wang, and A. Natarajan, "A 10GHz CMOS RX frontend with spatial cancellation of co-channel interferers for MIMO/digital beamforming arrays," in IEEE Radio Frequency Integrated Circuits Symp., May 2016, pp. 99-102.

[12] L. Zhang and H. Krishnaswamy, "A scalable $0.1-$ to- $1.7 \mathrm{GHz}$ spatiospectral-filtering 4-element MIMO receiver array with spatial notch suppression enabling digital beamforming," in IEEE Int. Solid-State Circuits Conf. (ISSCC) Dig. Tech. Papers, Jan. 2016, pp. 166-167.

[13] H. Krishnaswamy and L. Zhang, "Analog and RF Interference Mitigation for Integrated MIMO Receiver Arrays," Proc. IEEE, vol. 104, no. 3, pp. 561-575, March 2016.

[14] L. Zhang and H. Krishnaswamy, "A 0.1-to-3.1GHz 4-element MIMO receiver array supporting analog/RF arbitrary spatial filtering," in IEEE Int. Solid-State Circuits Conf. (ISSCC) Dig. Tech. Papers, Feb. 2017, pp. 410-411.

[15] L. Zhang, A. Natarajan, and H. Krishnaswamy, "Scalable Spatial Notch Suppression in Spatio-Spectral-Filtering MIMO Receiver Arrays for Digital Beamforming," IEEE J. Solid-State Circuits, vol. 51, no. 12, pp. 3152-3166, Dec. 2016.

[16] C. Kim, S. Joshi, C. M. Thomas, S. Ha, L. E. Larson, and G. Cauwenberghs, "A $1.3 \mathrm{mw} 48 \mathrm{mhz} 4$ channel mimo baseband receiver with 65 $\mathrm{db}$ harmonic rejection and $48.5 \mathrm{db}$ spatial signal separation," IEEE $J$. Solid-State Circuits, vol. 51, no. 4, pp. 832-844, April 2016.

[17] L. Zhang and H. Krishnaswamy, "Arbitrary Analog/RF Spatial Filtering for Digital MIMO Receiver Arrays," IEEE J. Solid-State Circuits, vol. 52, no. 12, pp. 3392-3404, Dec. 2017.

[18] S. Jang, J. Jeong, R. Lu, and M. P. Flynn, “A 16-Element 4-Beam 1 GHz IF $100 \mathrm{MHz}$ Bandwidth Interleaved Bit Stream Digital Beamformer in $40 \mathrm{~nm}$ CMOS," IEEE J. of Solid-State Circuits, vol. 53, no. 5, pp. 1302-1312, May 2018.
[19] S. Mondal and J. Paramesh, "A Reconfigurable 28-/37-GHz MMSEAdaptive Hybrid-Beamforming Receiver for Carrier Aggregation and Multi-Standard MIMO Communication," IEEE J. of Solid-State Circuits, vol. 54, no. 5, pp. 1391-1406, May 2019.

[20] S. Kalia, S. A. Patnaik, B. Sadhu, M. Sturm, M. Elbadry, and R. Harjani, "Multi-beam spatio-spectral beamforming receiver for wideband phased arrays," IEEE Trans. Circuits Syst. I, vol. 60, no. 8, pp. 2018-2029, Aug. 2013.

[21] S. Golabighezelahmad, E. Klumperink, and B. Nauta, "A 1-4 GHz 4x4 MIMO Receiver with 4 Reconfigurable Orthogonal Beams for Analog Interference Rejection," in IEEE Radio Frequency Integrated Circuits Symp. (RFIC), June 2019, pp. 339-342.

[22] J. Allen, "A theoretical limitation on the formation of lossless multiple beams in linear arrays," IRE Trans. on Antennas and Propag., vol. 9, no. 4, pp. 350-352, July 1961.

[23] J. Butler and R. Lowe, "Beamforming matrix simplifies design of electronically scanned antennas," Electronics Design, vol. 9, 1961.

[24] A. Tork and A. Natarajan, "Reconfigurable X-Band 44 Butler array in $32 \mathrm{~nm}$ CMOS SOI for angle-reject arrays," in IEEE MTT-S International Microwave Symp., May 2016, pp. 1-4.

[25] Z. Ru, N. A. Moseley, E. A. M. Klumperink, and B. Nauta, "Digitally Enhanced Software-Defined Radio Receiver Robust to Out-of-Band Interference," IEEE J. Solid-State Circuits, vol. 44, no. 12, pp. 33593375, Dec. 2009.

[26] D. Murphy, H. Darabi, A. Abidi, A. A. Hafez, A. Mirzaei, M. Mikhemar, and M. F. Chang, "A Blocker-Tolerant, Noise-Cancelling Receiver Suitable for Wideband Wireless Applications," IEEE Journal of SolidState Circuits, vol. 47, no. 12, pp. 2943-2963, Dec. 2012.

[27] C. Andrews and A. C. Molnar, "Implications of Passive Mixer Transparency for Impedance Matching and Noise Figure in Passive MixerFirst Receivers," IEEE Trans. Circuits Syst. I, vol. 57, no. 12, pp. 3092 3103, Dec. 2010.

[28] D. Yang, C. Andrews, and A. Molnar, "Optimized Design of N-Phase Passive Mixer-First Receivers in Wideband Operation," IEEE Trans. Circuits Syst. I, vol. 62, no. 11, pp. 2759-2770, Nov. 2015.

[29] "IRE Standards on Electron Tubes: Definitions of Terms, 1957," Proc.IRE, vol. 45, no. 7, pp. 983-1010, July 1957.

[30] S. Golabighezelahmad, E. Klumperink, and B. Nauta, "EVM-based Performance Evaluation of Co-channel Interference Mitigation using Spatial Filtering for Digital MIMO Receivers," accepted and to appear in the 2020 IEEE 92nd Vehicular Technology Conference (VTC2020Fall). 